\title{
Contacto precoz con la realidad asistencial: una experiencia piloto en medicina
}

\author{
J.E. Baños, M. Sentí, R. Miralles
}

Introducción. Varios estudios han sugerido que el contacto precoz del estudiante de medicina con la realidad asistencial puede tener unos efectos beneficiosos sobre su motivación, el conocimiento de la relación médico-paciente y la aceptación de la importancia de las materias médicas básicas. En el nuevo grado conjunto de Medicina de la Universitat Autònoma de Barcelona y la Universitat Pompeu Fabra se ha incorporado una asignatura denominada 'Prácticas de Grado' que se imparte durante los tres primeros años, destinada a permitir el contacto de los estudiantes con la asistencia primaria, los equipos de enfermería y los centros sociosanitarios. El presente artículo describe la experiencia y la opinión de los estudiantes que la cursaron durante el primer año.

Materiales y métodos. El artículo describe las características académicas de la asignatura. Se realizó una encuesta a los estudiantes al finalizar la asignatura para evaluar su grado de satisfacción y su percepción sobre el cumplimiento de los objetivos y las competencias que debían alcanzarse.

Resultados. Existió una elevada satisfacción con la nueva actividad y una percepción entre los estudiantes de que las competencias preestablecidas se habían alcanzado en su mayor parte.

Conclusión. El contacto precoz con la realidad asistencial es un elemento esencial para la comprensión de la actividad médica por los estudiantes de medicina de primer año.

Palabrasclave. Competenciastransversales. Contacto precoz. Educación médica. Profesionalismo. Técnicas de comunicación.

\section{Early contact with medical practice: a pilot experience in medical students}

Introduction. Several studies have suggested that early contact of medical students with medical care may have beneficial effects on their motivation, knowledge of doctor-patient relationship and acceptance of the interest of biomedical sciences. In the new joint degree of Medicine of Autonomous University of Barcelona and Pompeu Fabra University we have incorporated the subject 'Prácticas de Grado' during the first three years. It is devoted to permit the early contact of medical students with primary care, nursing teams and chronic care facilities. The present paper describes its academic features as well as the opinion of students during the first year of the experience.

Materials and methods. The characteristics of the subject are described in this section. A survey was performed in the students who completed the first year of the experience to get their degree of satisfaction and the perception with the achievement of pre-established competencies and educative objectives.

Results. Students were highly satisfied with the new subject and they believed that planned competencies and objectives were fully achieved in most of cases.

Conclusion. The early contact with health care is an important contribution to the understanding of the medical professions by medical students of first year.

Key words. Communication skills. Early contact. Generic competencies. Medical education. Professionalism.

\section{Introducción}

Tradicionalmente, el primer contacto de los estudiantes de medicina con los pacientes aparece en el tercer curso de sus estudios, cuando han adquirido una formación suficiente en las disciplinas básicas y entran en el período clínico de su formación, que incluye la fisiopatología y la semiología de las distintas enfermedades por aparatos y sistemas. Esta división entre el ciclo preclínico y clínico ha sido la norma durante la mayor parte del siglo xx [1]. No obstante, desde la publicación del informe del Ge-
Departamento de Ciencias Experimentales y de la Salud. Facultad de Ciencias de la Salud y de la Vida. Universitat Pompeu Fabra (J.E. Baños, M. Sentí) Departamento de Medicina. Unidad Docente del IMAS (UDIMAS-Hospital del Mar, Hospital de la Esperanza, Centro Fórum). Facultad de Medicina. Universitat Autònoma de Barcelona. Barcelona, España (R. Miralles).

Correspondencia: Dr. Josep Eladi Baños. Departamento de Ciencias Experimentales y de la Salud. Universitat Pompeu Fabra. Parc de Recerca Biomèdica de Barcelona. Doctor Aiguader, 88. E-08003 Barcelona.

E-mail:

josepeladi.banos@upf.edu

Agradecimientos

A los profesionales que han participado como tutores $y$ tutoras docentes en cada uno de los centros en donde se han realizado las prácticas: E. Gómez, A. Magán, A. Domínguez (Hospital del Mar); M. Carrasco, P. Navarro, M.J. Robles (Hospital de la Esperanza); A. Blanch, M. Rus, S. Moreno, M. Porta (CAP Larrard); J.M. Verdú, D. Martínez, M. Cabello (CAP Sant Martí); N. Calderón, N. Saavedra, C. Roqueta, B. González (Centro Fórum, Hospital del Mar). Su atención a los estudiantes y su colaboración en el proceso docente ha sido esencial para el éxito de la experiencia.

(c) 2011 Educación Médica 
Tabla I. Características y organización de la asignatura ‘Prácticas de Grado I'.

\begin{tabular}{ll}
\hline Carga académica & 4 ECTS a \\
\hline Actividad académica ${ }^{b}$ & Asistencia a centros sanitarios $(50 \mathrm{~h})$ \\
& Preparación del diario de prácticas (30 h) \\
& Redacción de un portafolio final $(20 \mathrm{~h})$ \\
\hline Duración & 5 h diarias durante los 10 días \\
\hline Centros & Asistencia primaria \\
& Unidades de hospitalización (atención hospitalaria) \\
& Centros sociosanitarios
\end{tabular}

a Cada crédito ECTS (European Credit Transfer System) equivale aproximadamente a $25 \mathrm{~h}$ de trabajo del alumno para conseguir las competencias (asistencia a los centros donde se realiza la práctica + tiempo de estudio + tiempo empleado en la realización de los diarios de prácticas y portafolios; total: 100 h); b En cada centro, los tutores fueron siempre profesionales que trabajaban en él (médicos o enfermeras). Cada tutor atendía simultáneamente a dos estudiantes como máximo. to una oportunidad extraordinaria de revisar en profundidad no sólo su estructura académica, sino también aspectos claves de la metodología docente y la adaptación a las nuevas necesidades sociales y profesionales. En el curso 2008-2009, la Universitat Autònoma de Barcelona (UAB) y la Universitat Pompeu Fabra (UPF) iniciaron un nuevo plan de estudios de Medicina de forma conjunta [11,12]. Entre las líneas maestras de dicho plan se encontraban cuatro objetivos generales: una formación intensa en las bases biológicas de la medicina, el desarrollo de una competencia clínica eficaz, la comprensión de las aspectos sociales y éticos de la profesión y una recuperación de la visión humanista de la medicina. Estos objetivos se encuentran básicamente recogidos en el concepto de profesionalismo médico ampliamente difundido en los últimos años y en la inclusión de los factores sociales dentro de la formación médica universitaria, siguiendo la estela de universidades de gran prestigio [13].

En el presente artículo se describen las características de una asignatura denominada 'Prácticas de Grado I' (PG-1), impartida durante el primer curso del grado de Medicina conjunto UAB-UPF. Así mismo, se describe también la opinión de los estudiantes sobre la asignatura.

eral Medical Council británico de 1993 [2] sos autores han defendido el interés de que los estudiantes tengan la oportunidad de conocer la realidad asistencial en fases precoces de su formación [3-8]. Las razones que justificarían tal opinión son diversas, pero se basan generalmente en la necesidad de que los futuros médicos conozcan los ámbitos donde se realiza la actividad clínica, los complejos procesos de la relación médico-paciente, las técnicas de comunicación y los condicionantes sociales que modelan la enfermedad, entre otros [9]. Así, una conferencia de consenso reciente ha sugerido que la experiencia temprana podría orientar los planes de estudio de medicina hacia el contexto social de la práctica médica, ayudaría a facilitar la transición al ambiente clínico, aumentaría la motivación de los estudiantes y facilitaría su interacción con los pacientes [10]. En el lado contrario, se puede abogar por seguir manteniendo la situación tradicional, debido a que las prácticas clínicas en los primeros años serían inadecuadas a causa de la escasa formación clínica de los estudiantes y, por ello, llevarían al desaprovechamiento de un tiempo que podría emplearse en otras actividades formativas. No sería ajeno al debate la necesidad de reducir la carga docente de algunas materias en los primeros años, una situación que puede crear conflictos en el ámbito universitario.

La adaptación de los planes de estudio al Espacio Europeo de Educación Superior (EEES) ha supues-

\section{Materiales y métodos}

\section{Entorno}

Los alumnos que participaron en la experiencia eran estudiantes de primer curso del grado conjunto de Medicina de la UAB-UPF, que se inició en el mes de septiembre de 2008 con 57 estudiantes. La dirección académica de este grado es responsabilidad de un consejo docente integrado de forma paritaria por profesores de ambas universidades y presidido de forma alterna, por períodos de dos años, por el decano de una de las dos facultades responsables de la docencia. En los meses siguientes, varios estudiantes solicitaron anulación de matrícula para trasladarse a otras universidades, con lo que el número real de estudiantes que iniciaron el curso fue de 53. La docencia está organizada por trimestres, con diez semanas de actividades docentes y dos dedicadas a la preparación y realización de exámenes. El primer trimestre finaliza en diciembre, el segundo a mediados de marzo y el tercero a mediados de junio.

\section{Descripción de la actividad}

La asignatura PG-1 pertenece al plan de estudios 
del grado conjunto que contiene otras dos asignaturas, denominadas 'Prácticas de Grado II y III', las cuales se imparten en el tercer trimestre de los tres primeros años, respectivamente. Tienen una carga académica de 4 ECTS y se realizan durante las tardes de dos semanas consecutivas en los meses de mayo y junio. Su horario es de 15:00 a 20:00 h de lunes a viernes. Se realizan en tres tipos de centros: centros de asistencia primaria, unidades de hospitalización en hospitales generales, y centros sociosanitarios. Está previsto que los estudiantes pasen por cada uno de los tres tipos de centros a lo largo de los tres primeros años, de manera que en el segundo y tercer curso, los estudiantes se incorporen a los centros en donde no habían estado en el curso anterior. En PG-1, los estudiantes asistieron a cada centro en grupos de diez alumnos como máximo, que se subdividieron a su vez en grupos de dos, supervisados cada uno de ellos por un tutor. Durante el período de prácticas, los estudiantes se integraron en los equipos asistenciales y observaron, participando cuando era posible, las tareas habituales de sus profesionales. Los estudiantes tuvieron también la oportunidad de observar las actividades de otros miembros de los equipos, como psicólogos, asistentes sociales o fisioterapeutas, en algunos de los centros. En las tablas I y II se resumen las características y organización de la asignatura, así como las competencias que se pretendían adquirir (tomadas del plan de estudios). Todos los tutores recibieron información específica sobre los objetivos educativos de la asignatura y una orientación detallada sobre las actividades específicas a realizar (Tabla III). La evaluación docente se realizó mediante un informe estandarizado para cada estudiante efectuado por el tutor responsable, la elaboración por cada alumno de un diario de prácticas y por un portafolio. En el primero se describían las actividades observadas y realizadas, así como todo aquello que se considerara oportuno reseñar. En el portafolio se describía lo que se había aprendido, las necesidades detectadas, una reflexión general sobre las prácticas y sugerencias para su mejora. Todo el proceso fue organizado y supervisado por tres coordinadores (los autores).

\section{Opinión de los estudiantes}

Los estudiantes finalizaron las actividades docentes previstas en la asignatura en el mes de junio. En septiembre se les invitó a contestar un cuestionario de forma voluntaria y anónima que incluía preguntas clasificadas en diversas categorías (Tabla IV):

- Preguntas en formato Likert (completamente de
Tabla II. Objetivos educativos y competencias de la asignatura 'Prácticas de Grado I'.

Objetivos educativos

1. Tomar contacto con la realidad asistencial antes de la formación clínica

2. Conocer las características del sistema sanitario y de los profesionales que intervienen

3. Establecer relación con el mundo de la asistencia clínica

4. Conocer los aspectos psicosociales vinculados a la salud (el estudiante ha de comprender la persona humana como un ser multidimensional, en el cual la interrelación de factores biológicos, psicológicos y sociales condicionan los estados de salud y enfermedad y sus manifestaciones)

5. Reflexionar sobre la complejidad de la relación entre pacientes y profesionales sanitarios (situaciones) problemáticas resultantes de los cambios sociológicos, demográficos y de los procesos migratorios)

Competencias

1. Conocer los fundamentos de la ética médica

2. Aplicar los valores personales y profesionales de excelencia, altruismo, sentido del deber, responsabilidad, integridad y honestidad en el ejercicio de la profesión

3. Conocer los aspectos de la comunicación en pacientes, familiares y su entorno social

4. Conocer los modelos de relación clínica (entrevista, comunicación verbal, comunicación no verbal) y sus interferencias

5. Comprender la importancia del trabajo en equipos multidisciplinares

acuerdo, de acuerdo, ni de acuerdo ni en desacuerdo, en desacuerdo, completamente en desacuerdo), donde se reformulaba una competencia prevista en el plan de estudios y de acuerdo con las obligatorias en el grado de Medicina (preguntas 1-5).

- Preguntas en formato Likert sobre la opinión de los estudiantes sobre diversas cuestiones, como decisión de dedicarse a la medicina, conocimiento de factores asociados a la enfermedad o contribución a la formación de los estudiantes (preguntas 6-8).

- Preguntas acerca del grado de satisfacción sobre diversos aspectos vinculados a las prácticas, puntuadas mediante una escala numérica de 1 (ninguna) a 10 (máxima) (pregunta 9).

\section{Análisis estadístico}

Se realizó un análisis estadístico descriptivo y los resultados se expresan como porcentajes, medianas o como la media \pm desviación estándar, según fueran variables discontinuas o continuas. Se realizó la comparación de los estudiantes que efectuaron sus prácticas en los diferentes centros mediante la $U$ de Mann-Whitney. No se realizaron comparaciones ni por género ni por edad debido a que la mayoría de los estudiantes eran mujeres y el intervalo de edad era muy estrecho. 
Tabla III. Actividades recomendadas para realizar en la asignatura 'Prácticas de Grado I' (el listado es orientativo y su realización dependió de cada situación).

Visita al centro asistencial

(destinado a cumplir los objetivos educativos 1-3 y las competencias 3-4 de la tabla II)

Conocer los circuitos de acceso de los pacientes y los familiares:

- En hospitales, ver urgencias, consultas externas, admisiones, espacios de exploraciones complementarias, salas de espera, plantas de hospitalización y comprender el circuito que sigue el paciente

- En centros de asistencia primaria, ver vestíbulo, salas de espera, consultas, otros espacios y entender el circuito que sigue el paciente

- En centros sociosanitarios, ver vestíbulo, salas comunes, consultas externas, espacios terapéuticos, hospital de día, salas de hospitalización de media y larga estancia, y entender el circuito que sigue el paciente

Conocimiento de la documentación clínica básica

(destinado a cumplir los objetivos educativos 1-3 y 5 y las competencias 1, 4 y 5 de la tabla II)

Conocer los principales documentos de la rutina asistencial:

- Historia clínica médica, de enfermería y de trabajo social

- Solicitudes de exploraciones complementarias e interconsultas a especialistas

- Documentación administrativa (identificación del enfermo)

- Otros documentos (consentimientos informados, voluntades anticipadas)

Comentar y reflexionar sobre la confidencialidad y el derecho a la privacidad del paciente

Entrevista con algún paciente (y/o familiar)

(destinado a cumplir los objetivos educativos 1 y 3-5 y las competencias 1-4 de la tabla II)

Entrevistar a un paciente (acompañados y/o supervisados por el tutor). En la entrevista debería salir la siguiente información:

- ¿Qué camino siguió el paciente a través del sistema sanitario hasta llegar allí?

- ¿Cuál ha sido su vivencia del proceso (¿satisfactoria?, ¿se ha sentido bien informado?)

- ¿Qué cambios ha provocado su enfermedad en su vida o en su entorno (repercusión psicosocial de la enfermedad, ¿cambios de residencia?, ¿dejar el trabajo?, ¿necesidad de cuidadores?)

- ¿Qué grado de participación ha tenido el paciente en las decisiones que se han tomado sobre su enfermedad?

Analizar las entrevistas desde el punto de vista de la 'comunicación'

(destinado a cumplir los objetivos educativos 1 y 5 y las competencias 1-4 de la tabla II)

Comentar aspectos relacionados con la comunicación con el paciente y/o familiar:

- ¿Nos hemos presentado?

- ¿Hemos explicado lo que vamos a hacer?

- ¿Nos hemos dirigido al paciente por su nombre?

- ¿Ha habido interrupciones o interferencias durante la entrevista?

- ¿Ha habido ambiente de privacidad?

Observación de las funciones de los diferentes profesionales sanitarios

(destinado a cumplir los objetivos educativos 2 y 5 y las competencias 2 y 5 de la tabla II)

Explicar las diferentes profesiones sanitarias y sus funciones:

- Enfermería

- Trabajo social

- Medicina

- Fisioterapia

Visitar y hablar con cada uno de estos profesionales y ver 'en directo' cómo ejercen sus funciones

Ver alguna actividad conjunta que se haga en equipo:

- Reuniones interdisciplinares

- Pases de visita conjuntos

- Sesiones

\section{Resultados}

\section{Descripción de la muestra}

De los 53 estudiantes del primer curso del grado, 46 $(88,6 \%)$ respondieron el cuestionario. De ellos, 35 $(76,1 \%)$ eran mujeres. La distribución por centros fue la siguiente: 14 realizaron las prácticas en centros de asistencia primaria, 14 en unidades de hospitalización y 18 en centros sociosanitarios.

\section{Opinión de los estudiantes sobre}

las competencias profesionales y otros aspectos asociados a la actividad médica

La tabla $\mathrm{V}$ recoge los resultados obtenidos en las respuestas de los estudiantes. En los ítems referidos a las competencias, las respuestas fueron muy similares, con la gran mayoría de los estudiantes respondiendo estar 'de acuerdo' o 'completamente de acuerdo'. Sólo en el ítem 4 un número significativo de estudiantes respondió la opción 'ni de acuerdo ni en desacuerdo' $(n=11)$, aunque la mediana del número de estudiantes se correspondió con la respuesta 'de acuerdo'.

En los ítems 6 a 8, una mayoría importante de estudiantes optaron por las opciones 'de acuerdo' o 'completamente de acuerdo'. En los dos primeros, referidos a su reafirmación de dedicarse a la medicina y a la comprensión de los factores asociados a la enfermedad, la mediana correspondió a la opción 'de acuerdo', mientras que en el último, referido a la contribución de las prácticas para su formación, la mediana se situó en 'completamente de acuerdo', con un $97,8 \%$ de estudiantes favorables a esta opción.

\section{Satisfacción de los estudiantes con las prácticas}

La tabla VI recoge los resultados de las medias de las puntuaciones (de 1 a 10), referidos a la satisfacción con diversos aspectos vinculados a la realización de las prácticas. Como puede observarse, a excepción de la valoración del horario de las prácticas (puntuación media: 6,7) y de su organización (puntuación media: 7,2), la mayoría de ítems fueron puntuados con un valor medio igual o superior a 8 . En este sentido, destacan especialmente los ítems 'experiencia vital' $(9,1)$, 'relación con los pacientes' $(8,6)$ y 'atención con tutores' $(8,8)$.

\section{Comparación de los resultados por centro}

No se observaron diferencias significativas al anali- 
zar los resultados de cada ítem en función del centro donde se realizaron las prácticas.

\section{Discusión}

Los resultados del presente estudio muestran que los estudiantes de medicina tienen una opinión muy satisfactoria de la experiencia presentada en este artículo. Asimismo, consideraron que las competencias que se habían vinculado a la realización de la asignatura PG-1 se habían alcanzado de forma satisfactoria. Destaca además su elevada satisfacción con algunos aspectos asociados a su profesión, como la relación con los pacientes. Especialmente relevante fue su consideración de las prácticas como una experiencia vital.

La experiencia del contacto precoz de los estudiantes con el medio asistencial no es algo nuevo. En este sentido, y de formas diversas, ya ha sido utilizada por otras universidades como Sherbrooke [14], Leeds [15], Aarhus [16], Malmö [9], Otago [17], Linköping [18], Heidelberg [19], Natal [20], Glasgow [21], East Anglia [22] y Götheborg [23]. De hecho, la mayoría de las universidades británicas [22] y suecas [23] incluyen tales actividades en sus planes de estudio.

Existen pocos antecedentes en nuestro medio de una experiencia similar a la que describimos en este artículo. Por ejemplo, en la Licenciatura de Medicina impartida en la UAB se realiza una experiencia pionera similar con una asignatura llamada 'Introducción a la Medicina' (plan de estudios de 2002), dentro de la cual existe un módulo denominado 'Practicum de introducción a la medicina comunitaria' [24]. En esta asignatura, los estudiantes de primer curso realizan una estancia con médicos de asistencia primaria. No obstante, su duración es más corta que la descrita aquí y no incluye periodos de estancia en unidades hospitalarias ni en centros sociosanitarios. Desconocemos si otras facultades de medicina españolas organizan estancias similares para sus estudiantes en los primeros años de sus estudios. Tales actividades serían muy útiles para introducir a los alumnos de forma muy precoz en realidades que serían difíciles de comprender de otra manera y aumentar su motivación a lo largo de sus estudios. Estos dos aspectos merecen, en nuestra opinión, un comentario más detallado.

Es habitual que los planes de estudio de Medicina no consideren las prácticas en el medio asistencial como mínimo hasta tercer curso y, en este caso, para la enseñanza de la anamnesis y la exploración física de forma casi exclusiva. En los años siguientes las
Tabla III. Actividades recomendadas para realizar en la asignatura 'Prácticas de Grado I' (el listado es orientativo y su realización dependió de cada situación) (cont.).

Observación de actividades profesionales

(destinado a cumplir los objetivos educativos 1 y 3 y las competencias 2, 4 y 5 de la tabla II)

Estar presentes y ver cómo se hace una anamnesis y una exploración física

Estar presentes y ver cómo se hace una toma de constantes, determinación de peso y talla $u$ otras medidas habituales

Ver cómo se hacen algunas técnicas básicas de cuidados:

- Movilizaciones

- Curas de heridas

- Administración parenteral de medicamentos y de colirios

- Colocación de sondas

Observación de informes y sistemas de informaciónentre profesionales y diferentes niveles asistenciales (destinado a cumplir los objetivos educativos 1-3 y 5 y las competencias 4 y 5 de la tabla II)

Ver informes y sistemas de información clínica de un paciente:

- Entre traslados de centros

- Cuando es enviado de un profesional a otro

Conocer los sistemas de comunicación entre los diferentes profesionales sanitarios u otros implicados en la atención de los pacientes

Tabla IV. Ítems del cuestionario de evaluación de la asignatura 'Prácticas de Grado I', que se utilizó para evaluar la opinión de los estudiantes.

1. Las prácticas me han permitido conocer los fundamentos de la ética médica (competencia 1)

2. Las prácticas me han permitido conocer los valores personales y profesionales de excelencia, altruismo, sentido del deber, responsabilidad y honestidad en el ejercicio de la profesión (competencia 2)

3. Las prácticas me han permitido conocer los aspectos de la comunicación con pacientes, familiares y sus entornos sociales (competencia 3)

4. Las prácticas me han permitido conocer los modelos de relación clínica (entrevista, comunicación verbal, comunicación no verbal) y las interferencias (competencia 4)

5. Las prácticas me han permitido comprender la importancia del trabajo en equipos multidisciplinarios (competencia 5)

6. Después de realizar estas prácticas considero que mi decisión de estudiar medicina se ha reforzado

7. Después de realizar estas prácticas comprendo mucho mejor los factores asociados a la enfermedad y las necesidades de los pacientes

8. Considero que las prácticas son una contribución importante a la formación de los estudiantes de medicina

9. Grado de satisfacción con los aspectos siguientes:

- Organización

- Horario

- Relación con los coordinadores

- Relación con los pacientes

- Aprendizaje

- Experiencia vital

- Comprensión de la profesión médica

- Atención de los tutores

- Conocimiento de los centros asistenciales

- Conocimiento de los procesos asistenciales

Preguntas 1 a 8: respuestas tipo Likert (completamente de acuerdo, de acuerdo, ni de acuerdo ni en desacuerdo, en desacuerdo, completamente en desacuerdo). Pregunta 9: respuestas en forma de escala numérica, de 1 (nada) a 10 (mucho). 
Tabla V. Opiniones de los estudiantes sobre las competencias profesionales y otros aspectos asociados a las actividades llevadas a cabo durante las prácticas (estudiantes que respondieron a cada opción, $n=46$ ).

\begin{tabular}{|c|c|c|c|c|c|c|c|}
\hline Ítem & Descripción & A & B & C & D & $\mathrm{E}$ & Mediana \\
\hline 1 & $\begin{array}{l}\text { Competencia } 1 \\
\text { Valores personales y profesionales }\end{array}$ & $18(39 \%)$ & $27(58,7 \%)$ & $1(2,2 \%)$ & - & - & De acuerdo \\
\hline 2 & $\begin{array}{l}\text { Competencia } 2 \\
\text { Aspectos de comunicación }\end{array}$ & $33(71,7 \%)$ & $13(28,3 \%)$ & - & - & - & $\begin{array}{l}\text { Completamente } \\
\text { de acuerdo }\end{array}$ \\
\hline 3 & $\begin{array}{l}\text { Competencia } 3 \\
\text { Modelos de relación clínica }\end{array}$ & $19(41,3 \%)$ & $26(56,5 \%)$ & $1(2,2 \%)$ & - & - & De acuerdo \\
\hline 4 & $\begin{array}{l}\text { Competencia } 4 \\
\text { Fundamentos de la ética médica }\end{array}$ & $7(15,2 \%)$ & $28(60,9 \%)$ & $11(23,9 \%)$ & - & - & De acuerdo \\
\hline 5 & $\begin{array}{l}\text { Competencia } 5 \\
\text { Trabajo en equipos multidisciplinares }\end{array}$ & $20(43,5 \%)$ & $23(50 \%)$ & $3(6,5 \%)$ & - & - & De acuerdo \\
\hline 6 & Reafirmación de dedicarse a la medicina & $22(47,8 \%)$ & $21(45,7 \%)$ & $1(2,2 \%)$ & $1(2,2 \%)$ & $1(2,2 \%)$ & De acuerdo \\
\hline 7 & $\begin{array}{l}\text { Comprensión de los factores asociados } \\
\text { a la enfermedad }\end{array}$ & $14(30,4 \%)$ & $25(54,3 \%)$ & $6(13,0 \%)$ & $1(2,2 \%)$ & - & De acuerdo \\
\hline 8 & Contribución importante a la formación & $37(80,4 \%)$ & $8(17,4 \%)$ & $1(2,2 \%)$ & - & - & $\begin{array}{l}\text { Completamente } \\
\text { de acuerdo }\end{array}$ \\
\hline
\end{tabular}

A: completamente de acuerdo; B: de acuerdo; C: ni de acuerdo ni en desacuerdo; D: en desacuerdo; E: completamente en desacuerdo.

prácticas clínicas se realizan en diversas especialidades para profundizar en estos elementos y aprender las características de las entidades clínicas más frecuentes en cada una de ellas. Es mucho menos frecuente que se preste una atención más detallada a los aspectos psicosociales asociados a la enfermedad, la actuación multidisciplinaria o los compromisos asociados a la profesión médica. La mayoría de éstos son atendidos en el último curso durante las prácticas de rotatorio y de forma completa durante el período de especialización. Con ello se mantiene un modelo de aprendizaje basado de forma preferente en la instrucción jerárquica, que tiene menos oportunidades de crear profesionales críticos que puedan introducir cambios sustanciales en las dinámicas asistenciales cuando en el futuro tengan la oportunidad de hacerlo. Una visión global de la atención sanitaria, desde los equipos de medicina primaria a los propios de los centros sociosanitarios, pasando por los equipos hospitalarios de enfermería, puede contribuir a un conocimiento más holístico de las realidades asistenciales, más allá de la formación 'hospitalocéntrica' que ha sido la habitual en los tiempos más recientes. La contribución de los médicos de asistencia primaria a la formación de los estudiantes de medicina es cada vez más valorada [23], incluso cuando éstos tienen una experiencia clínica mínima [25]. La necesidad de que los estudiantes tengan experiencias en diversos ámbitos sanitarios ha sido reconocida por la propia World Federation of Medical Education [4]. Además, la inclusión de un período de prácticas en unidades de hospitalización, tutorizado por equipos de enfermería, algo muy poco habitual en nuestro medio, debería contribuir a la comprensión del trabajo de profesionales que van a compartir espacios y actuaciones en muchos de los ámbitos de la vida profesional de los médicos.

El segundo aspecto es el de la motivación. Para Flórez [26], la motivación es el principal elemento que guía nuestra conducta, y lo identifica como el deseo de alcanzar lo que se nos representa como agradable y la evitación de lo que se considera desagradable. En el caso de la docencia de la medicina, la intensidad y la orientación de las disciplinas básicas, absolutamente necesarias para la formación científica del médico, son habitualmente malentendidas y no es infrecuente que los estudiantes las consideren como un obstáculo que debe franquearse para llegar a las asignaturas clínicas, que son las que han motivado la elección de sus estudios en la mayoría de ellos. La inclusión de asignaturas que ayuden en estos primeros años a satisfacer la curio- 
sidad de los estudiantes en el ámbito clínico, y que les aporten elementos de comprensión de la profesión médica, debe ser bienvenida. Como se respondía en uno de los ítems del cuestionario, los estudiantes manifestaron de forma mayoritaria que las prácticas habían contribuido a reafirmar su decisión de dedicarse a la medicina.

Otro aspecto que merece la pena destacar es la contribución de las prácticas a alcanzar determinadas competencias que pueden ser difíciles de completar en otras asignaturas. Entre éstas se encuentran los valores profesionales, los aspectos comunicativos, los modelos de relación clínica o el trabajo de equipos multidisciplinares. En todas ellas, los estudiantes percibieron que PG-1 contribuyó de manera positiva a comprender tales procesos. La lectura de sus diarios de prácticas y portafolios muestra que la aprehensión de tales competencias fue notablemente elevada, lo que refuerza el interés de continuar con tales actividades. Estos resultados coinciden con los observados por otros autores, en los que los estudiantes señalaron la utilidad de la experiencia para aprender aspectos como la percepción de la enfermedad por los pacientes, la necesidad de comunicarse de forma clara, la interacción médico-paciente, el significado de la actividad médica, así como para aumentar la motivación por sus estudios [14,15,20,22,23,27-29]. En este sentido, Johnson y Scott [30] describieron la mayor satisfacción de los estudiantes que participaron en actividades de contacto precoz con pacientes sobre sus estudios médicos que los que no las realizaron. Para otros, el contacto clínico precoz supuso una percepción de los aspectos más humanísticos de la práctica médica [20] y la concepción del paciente de forma mucho más integral [31]. Asimismo, los estudiantes consideran que la posibilidad de tener un contacto con los pacientes en los años iniciales del plan de estudios, tanto en el ámbito hospitalario como comunitario, es especialmente importante para su formación [21]. Finalmente, la experiencia contribuye a que los estudiantes tengan la percepción de la importancia de las ciencias básicas para su práctica futura [22,23,32]. Parece, además, que el contacto clínico precoz puede mejorar la percepción de los estudiantes del conocimiento teórico, por lo que numerosas facultades de medicina han revisado sus planes de estudios para permitir una integración más vertical entre las ciencias básicas y las clínicas $[17,23]$.

Existen opiniones críticas que cuestionan el nivel de aprovechamiento del tiempo de los estudiantes en estas prácticas tan precoces, especialmente en cuanto a la adquisición de conocimientos
Tabla VI. Grado de satisfacción con diversos aspectos asociados a las prácticas, evaluadas con una escala numérica de 1 (ninguna satisfacción) a 10 (máxima satisfacción). Los valores se expresan como media \pm desviación estándar.

\begin{tabular}{lc}
\hline Organización de las prácticas & $7,2 \pm 1,6$ \\
\hline Horario de las prácticas & $6,7 \pm 1,6$ \\
\hline Relación con los coordinadores & $8,0 \pm 1,3$ \\
\hline Relación con los pacientes & $8,6 \pm 0,9$ \\
\hline Grado de aprendizaje & $8,1 \pm 1,3$ \\
\hline Prácticas como experiencia vital & $9,1 \pm 0,9$ \\
\hline Comprensión de la profesión médica & $8,0 \pm 1,2$ \\
\hline Atención de los tutores & $8,8 \pm 0,9$ \\
\hline Conocimiento de los centros asistenciales & $8,0 \pm 0,9$ \\
\hline Conocimiento de los procesos asistenciales & $8,0 \pm 0,7$ \\
\hline
\end{tabular}

teórico-prácticos, ya que alumnos de primer y segundo curso, que todavía no tienen los conocimientos básicos de anamnesis, ni semiología, ni fisiopatología, podrían no llegar a comprender los mecanismos y las causas de lo que están viendo, siendo éste un motivo frecuente por el que algunos profesores con experiencia se muestran escépticos en la aceptación de estas prácticas [9]. Cabría recordar, en este sentido, que dicho escepticismo está frecuentemente motivado por una confusión en el verdadero objetivo de las prácticas. Éste no es el aprendizaje del manejo clínico del paciente (diagnóstico, exploraciones complementarias, tratamientos), sino que consiste, tal y como se ha mencionado, en una introducción general al funcionamiento del sistema sanitario y de sus profesionales. En este sentido, es innegable que el aprendizaje por observación directa en un escenario real ejerce un impacto superior al de otros tipos de docencia, por lo que habrá que ver si las observaciones clínicas de estos estudiantes les permitirá aprender y comprender con más facilidad los procesos clínicos y las enfermedades que estudiarán en futuros curso venideros. Esta suposición está refrendada por experiencias previas [27]. Otros autores han mostrado, comparando su grupo con controles históricos, que el contacto precoz puede mejorar las actitudes de los estudiantes hacia, por ejemplo, los pacientes ancianos [17]. 
Como cualquier otra actividad, tampoco la presente se encuentra desprovista de problemas. Aunque en nuestro caso fue muy positiva, otros autores han citado diversos aspectos que pueden amenazarla [9]. Entre ellos se encuentran la falta de tiempo, la escasa experiencia clínica de los estudiantes, la alteración eventual de la relación médico-enfermo y la dificultad para dar el adecuado feedback a los estudiantes. Estudios realizados en Gran Bretaña también señalan la presión asistencial, la falta de tiempo y el espacio reducido disponible en las consultas como los principales obstáculos para la docencia de los estudiantes, sobre todo en asistencia primaria [33-35]. El aumento de la carga de trabajo también se señaló como un obstáculo en un estudio realizado en Suecia [23]. Estos aspectos pueden ser relevantes y se deben tener en cuenta cuando se planifiquen actividades docentes de este tipo, para evitar un número excesivo de estudiantes por tutor, la aceptación de los estudiantes de que deberán abandonar la consulta cuando sea necesario o la asunción por los médicos de la limitada o nula experiencia clínica de los estudiantes.

Las conclusiones de este estudio deben contemplarse en función de sus propias limitaciones. En primer lugar, se trata de la evaluación subjetiva de los estudiantes de la actividad y, por tanto, una impresión de estudiantes que acaban de ingresar en la universidad. En segundo, se trata de una primera experiencia que debe tener continuidad y de la que se tendrá una opinión más definitiva al finalizar los tres años. En tercero, los estudiantes sólo tuvieron experiencia en uno de los tres tipos de centros escogidos. Estas limitaciones son, en nuestra opinión, inevitables al considerar un estudio piloto como el que se describe, pero consideramos que la opinión de los estudiantes sobre su formación puede dar importantes pistas para conocer su percepción sobre la asunción de los objetivos preestablecidos y cómo contribuir a mejorarla.

Pese a todo lo expuesto, la cuestión central es si el contacto precoz con el medio asistencial es un elemento claramente positivo para la formación médica, más allá de impresiones subjetivas. En este sentido, Dornan et al [36] han realizado una revisión sistemática de los resultados de tal experiencia en la bibliografía médica. Sus conclusiones, tras analizar 73 estudios empíricos sobre este tema, son que la experiencia precoz ayuda a que los estudiantes adquieran numerosos objetivos afectivos, como la empatía hacia los pacientes y la construcción de una autoconciencia, y contribuye a su satisfacción con el plan de estudios. Además, les da mayor confianza en su relación con los pacientes y permite comprender cómo funciona el sistema sanitario y las técnicas básicas de comunicación y de exploración [36]. La mayoría de estos ítems pueden incluirse dentro del profesionalismo, un importante concepto vinculado a cómo ejercer la actividad médica en el siglo XXI [37-39].

En resumen, la experiencia de PG-1 fue altamente satisfactoria y creemos, como los estudiantes, que puede ser una contribución importante a la formación de los futuros médicos en aspectos básicos que mejoren su percepción del profesionalismo. Como ha escrito Válková [31], los estudiantes aprenderán más fácilmente que el conocimiento del paciente sólo en términos físicos apenas contribuirá a eliminar todos sus problemas de salud.

Bibliografía

1. Dornan T. Osler, Flexner, apprenticeship, and 'the new medical education. J Royal Soc Med 2005; 98: 91-5.

2. General Medical Council. Tomorrow's doctors: recommendations on undergraduate medical education. London: General Medical Council; 1993.

3. Högskoleverket (Agencia Nacional para la Educación Superior). Läkarutbildningen I Sverige -Hur Bra Är Den? (Rapport 1997; 27R). Stockholm: Högskoleverket; 1997.

4. World Federation for Medical Education. International standards in medical education: assessment and accreditation of medical schools' educational programmes. Med Educ 1998; 32: 549-58.

5. General Medical Council. Tomorrow's doctors. 2 ed. London: General Medical Council; 2002.

6. Goic A. Seminario sobre formación de médicos en la actualidad en Chile. Rev Med Chile 2003; 131: 209-12.

7. Alfonso MT, Carrasco M, Escanero J, Fonseca M, Gual A, Manso JM, et al. Recomendaciones para un nuevo proceso de reforma curricular en las facultades de medicina españolas. Educ Med 2005; 8 :3-7.

8. Rozman C. Reflexiones sobre la universidad en el ámbito biomédico. Lección Pi i Sunyer, 2005, Facultad de Medicina de la Universidad de Barcelona. Educ Med 2005; 8: 183-90.

9. Haffling AC, Hakansson A, Hagander B. Early patient contact in primary care: a new challenge. Med Educ 2001; 35: 901-8.

10. Dornan T, Bundy C. What can experience add to early medical education? Consensus survey. BMJ 2004; 329: 834-7.

11. Plan de estudios del Grado de Medicina UAB-UPF. URL: http://www.upf.edu/estudiants/titulacions/grau-medicina/ $\mathrm{pla} / .[05.04 .2010]$.

12. Plan de estudios del Grado de Medicina UAB-UPF. URL: http://www.uab.es/servlet/Satellite/estudiar/tots-els-estudis/ informacio-general/medicina-grau-uab/upf-1089963374371. $\mathrm{html}$ ?param1 $=1216620146538 \&$ param $11=6$. $[05.04 .2010]$.

13. Wiener CM, Thomas PA, Goodspeed E, Valle D, Nichols DG. From 'Genes to Society' - the logic and process of the new curriculum for the Johns Hopkins University School of Medicine. Acad Med 2010; 85: 498-506.

14. Barbeau D, Quesnel J, Des Marchais B. Students' perceptions after one year of problem-based learning at Sherbrooke. Ann Comm Oriented Educ 1990; 3: 25-33.

15. Cade J. An evaluation of early patient contact for medical students. Med Educ 1993; 27: 205-10.

16. Eika B, Molgaard H, Sonne O, Jorgenson JO. Early clinical exposure -an instant success. The new medical curriculum at the University of Aarhus. Ugeskr Laeger 2001; 163: 3626-9.

17. Wilkinson TJ, Gower S, Sainsbury R. The earlier, the better: 
the effect of early community contact on the attitudes of medical students to older people. Med Educ 2001; 36: 540-2

18. Dahle LO, Brynhildsen J, Behrbohm Fallsberg M, Rundquist I, Hammar M. Pros and cons of vertical integration between clinical medicine and basic science within a problem-based undergraduate medical curriculum: examples and experiences from Linköping, Sweden. Med Teach 2002; 24: 280-5.

19. Wiesemann A, Engeser P, Barlet J, Muller-Buhl U, Szecsenyi $\mathrm{J}$. What students and teaching doctors in Heidelberg think about early patient contact and tasks in general practice. Gesundheitswesen 2003; 65: 572-8.

20. McLean M. Sometimes we do get it right! Early clinical contact is a rewarding experience. Educ Health 2004; 17: 42-52.

21. Goldie J, Dowie A, Cotton P, Morrison J. Teaching professionalism in the early years of a medical curriculum: a qualitative study. Med Educ 2007; 41: 610-7.

22. Howe A, Dagley V, Hopayian K, Lillicrap M. Patient contact in the first year of basic medical training. Feasible, educational, acceptable? Med Teacher 2007; 29: 237-45.

23. Von Below B, Hellquist G, Rödjer S, Gunnarson R, Björkelund C, Wahlqvist M. Medical students' and facilitators' experiences of an early professional contact course: active and motivated students, strained facilitators. BMC Med Educ 2008; 8: 56

24. Introducció a la Medicina: Practicum d'introducció al sistema sanitari i a l'atenció de salud. URL: http://www. uab.cat/medicina. URL: http://www.uab.cat/servlet/Satellite/ estudiar/titols-a-substituir-per-graus/pla-d-estudis1232609138639.html?param1=1089612449066. [05.04.2010]

25. Kendrick T, Freeling P. A Communications skills course for pre-clinical students. Med Educ 1993; 27: 211-7.

26. Flórez J. Cerebro: el mundo de las emociones y de la motivación. In Mora F, ed. El cerebro íntimo. Barcelona: Ariel Neurociencia; 1996. p. 165-85.

27. Briggs-Style C, Maxwell JA, Moore GT. The effects of early patient contact: the student's perspective. Acad Med 1990; 65 (Suppl): S33-4.

28. Wykurz G, Kelly D. Developing the role of patients as teachers: a literature review. BMJ 2002; 325: 818-21.

29. Littlewood S, Ypinazar V, Margolis S, Scherpbier A, Spencer S, Dornan T. Early practical experience and the social responsiveness of clinical education: systematic review. BMJ 2005; 331: 387-91.

30. Johnson AK, Scott CS. Relationship between early clinical exposure and first-year students' attitudes toward medical education. Acad Med 1998; 73: 430-2.

31. Válková L. First early patient contact for medical students in Prague. Fam Pract 1997; 14: 394-6.

32. Kidd J, Patel V, Peile E, Carter Y. Clinical and communications skills need to be learnt side by side. BMJ 2005; 330: 374-5.

33. Fine B, Seabrook M. GP's attitudes towards increased medical education in the community. Educ Gen Pract 1996; 7: 42-7.

34. Hartley S, Macfarlane F, Gantley M, Murray E. Influence on general practitioners of teaching undergraduates: qualitative study of London general practitioners teachers. BMJ 1999; 319: 1168-71.

35. Gray J, Fine B. General practitioners teaching in the community: a study of their teaching experience and interest in undergraduate teaching in the future. Br J Gen Pract 1997; 47: 623-6.

36. Dornan T, Littlewood S, Margolis SA, Scherpbier A, Spencer J, Ypinazar V. How can experience in clinical and community settings contribute to early medical education? A BEME systematic review. Med Teach 2006; 28: 3-18.

37. Irvine $\mathrm{D}$. The performance of doctors: new professionalism. Lancet 1999; 353: 1174-7.

38. Medical Professionalism Project. Medical professionalism in the new millennium. Clin Med JRCPL 2002; 2: 116-8.

39. Pardell-Alentá $\mathrm{H}$. The physician of the future/El médico del futuro. Barcelona: Fundación Educación Médica; 2009. 